\title{
Levels of intelligent information access using ontology transformations
}

\author{
Lena Wenner $^{1 *}$, and Nergis Kuru ${ }^{1 * *}$ \\ ${ }^{1}$ Karlsruhe University of Applied Sciences, Faculty of Information Management and Media, 76133 Karlsruhe, Germany
}

\begin{abstract}
Current techniques of intelligent information access have surpassed the mere provision of static information. They attempt to overcome the limitations of traditional content delivery processes by exploiting information retrieval techniques. While the existing literature has extensively explored the adoption of these techniques, comparatively little notice has so far been taken of their application as ontology transformations. This paper aims to explore levels of intelligent information access from a practical perspective. Applying the theoretical framework of information retrieval techniques, we identify and define levels of information access and propose respective ontology transformations. For this purpose, we decided to use an ontologyediting tool for modeling, transforming and displaying information about the domain of Smart Homes.
\end{abstract}

\section{Introduction}

Classic technical documentation still considers end-user products to be manufactured for a specific purpose and used in predefined and predictable situations. This understanding also applies to the rather static and extensive information products. However, as Leukert [1] points out, the expectations of users for the right amount of personalized, situation-dependent and contextual information are increasing. The approaches of the Internet of Things (IoT) and Industry 4.0 are changing the context in which (technical) information is embedded, accessed and used. The context can no longer be predefined, but rather evolves while using the products $[2,3]$.

Considering the paradigm shift from static information in classic manuals to on-demand delivery of information, the provision of intelligent information is becoming a fundamental requirement. In the context of this paper, the term intelligent information is understood as information that can be requested, processed and delivered both manually and automatically according to specific requests and use cases [4]. Information retrieval techniques evolved to give principled approaches to accessing intelligent information. These techniques aim at providing users with content that is both relevant and intelligible for them and their needs [5]. They can be brought into the field of ontologies to tackle the aforementioned challenges.

The existing literature has extensively explored the adoption and implementation of information retrieval techniques that have become the preferred form of information access [5]. However, our review of previous studies also suggests that the application of these techniques in the context of ontologies and ontology transformations is a topic that has not been sufficiently covered in the field of technical communication. Thus, the primary goal of this paper is to explore and compare different retrieval techniques from a practical perspective of both technical writers and ontology developers. To position our findings, we tailor these techniques to use cases and implement them as ontology transformations. By applying the latter to an ontology model, we propose opportunities to enhance the delivery of intelligent information and its access.

The structure of this paper is as follows: Section 2 is devoted to the basics of ontologies and ontology transformations. It also presents our research approach. Section 3 provides definitions of different levels of intelligent information access, combined with information retrieval techniques. Here, the focus lies on how these retrieval techniques were designed and implemented as ontology transformations. In section 4 we discuss the limitations of this study. Finally, section 5 reviews the main findings and provides a conclusion.

\section{Background}

Various authors have been discussing the many discourses and explanations for the term ontology. Guarino and Giaretta [6] even devote an entire paper to its terminological clarification and provide a list of several possible interpretations. These divergences in discourse suggest that the precise meaning of the term depends on the understanding of the disciplines in which ontologies are used. For this study, ontologies are defined as conceptualizations of knowledge and explicit

\footnotetext{
Corresponding authors:

*Lena Wenner: lena.wenner@gmx.de,

${ }^{* * *}$ Nergis Kuru: nergis.k@live.de
} 
specifications of terms in a domain of interest (also domain of discourse) [7].

Ontologies are to model semantic networks and complex relations to enable a mutual understanding of the meaning of specific vocabulary terms and to facilitate information integration [8] across information sources. As such, they differ from existing approaches and methods like mind maps, thesauri, and hierarchical taxonomies.

Gruber [7] provides a detailed account of ontologies' nature. He explains that ontologies can be formally described by a set of objects (e.g. classes, instances, and relations) which are reflected in representational vocabularies, and which may differ depending on the ontology-editing tools used to represent the concepts of a domain and the relations among them. Given this explanation, ontologies are unambiguously formalized in logic-based representational vocabularies. Thus, their syntax and semantics are machine-interpretable that enables querying, logic-based reasoning [7], and "sensemaking" in pursuit of meaningful knowledge. These mechanisms can be used to enhance more intelligent information search, delivery and access processes.

The representational vocabularies, their syntax and semantics form the backbone of ontology transformations. The meaning of the latter can be very broad. A more general definition by the Cambridge Dictionary considers transformations as a change in the appearance or character of something to improve itself in any way [9]. This is also the case in ontology transformations. As defined, they perform modifications on the formal semantics and ontology patterns, transforming (parts of) an ontology from one modeling choice to another. They do so by taking transformation instructions (specified as a set of rules or procedures) and an ontology as an input. An advantage worth noting is that the same conceptualization can be modeled and transformed in various ways [10].

The main purpose of ontology transformations is to convert information contained in ontologies into useful knowledge and valuable new insights. To conduct this, we decided to use the web-based tool Ontolis for modeling, displaying and transforming information about Smart Homes, which is the domain of discourse in this scenario. Consequently, the terminology we use to describe the practical implementation is ontolis-specific (terms are emphasized). While any ontology-editing tool could have been used, we chose Ontolis because it accommodates state-of-the-art semantic modeling and knowledge representation. Also, it supports sophisticated ontology transformations at different stages. For a profound explanation of the modeling methodology and the development practices in Ontolis, the reader is referred to a case study by Fischer and Krägel [11], which we deemed outside the scope of this paper.

\subsection{Research approach}

Ontology transformations strongly rely on the modeling of ontologies. Thus, the first steps of this research were to define the domain of discourse and develop specific use cases that guided many of the modeling decisions. These steps also had a significant impact on the application of the ontology transformations.

The ontology we developed for Smart Homes is intended to support end-users in retrieving high-level information on smart devices, e.g. a smart thermostat, smart lighting or smart speaker. For this projected task, we defined use cases according to the framework offered by Cockburn [12]. They go beyond single-session lookup tasks and consider more complex queries (such as in "Which smart devices are compatible with my existing ones controlled by the Apple Home Kit System?"). While defining a clear scope of information search and access scenarios, the use cases also provided the necessary scaffolding to develop the ontology.

Figure 1 presents an extracted part of the ontology in Ontolis. It consists of various components (represented as nodes) that constitute the semantic model and the information corpus for Smart Homes.

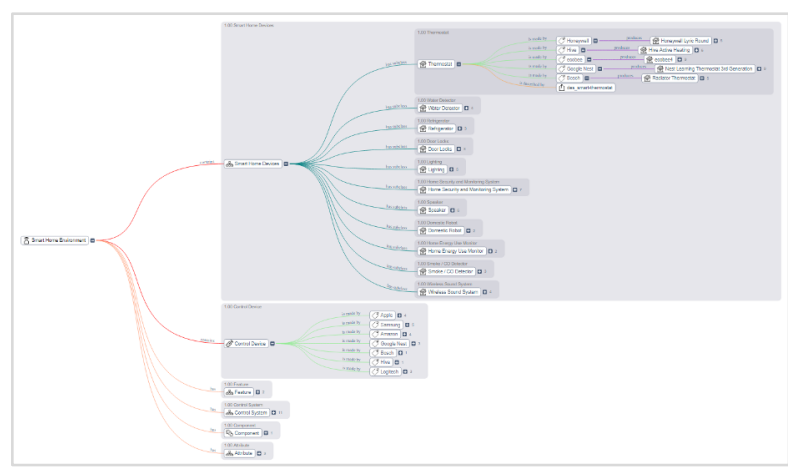

Fig. 1 Extracted part of the ontology model for Smart Homes developed in Ontolis.

The ontology model is not comprehensive in covering all possible topics and devices related to the domain of Smart Homes. However, it sufficiently describes and represents the classes, instances, attributes, and relations, which served as a suitable base for the ontology transformations.

\section{Levels of intelligent information access}

Intelligent information access attempts to overcome the limitations of current information delivery processes by exploiting information retrieval techniques. The latter normally use (direct or indirect) inputs and facilitate search and decision processes [13] to match information items from among large collections to the request [5]. This activity involves evaluating and weighting the information "so that each user can quickly find information that is both relevant and comprehensive for their needs." [5]

In the following, we define and summarize four levels of intelligent information access in the context of ontologies. We only focus on the fundamental notions needed for later references in the sections where ontology transformations are described. 


\subsection{Level 1: filtering}

In general terms, filtering is understood as the removal of information rather than finding relevant content in a set of data. In this case, users are only provided with information that is not removed [14].

Although various filtering techniques share the goal of delivering only relevant information to users, we focus on rule-based filtering systems. The filtering component of those systems consists of a set of rules that are applied for information to be filtered. They instruct the filtering component to either filter out the information or to treat it as relevant as soon as the conditions of a rule are met [14]. The same applied to ontologies, their filtering components delete non-relevant information without changing the original structure of the ontology (see Figure 2).
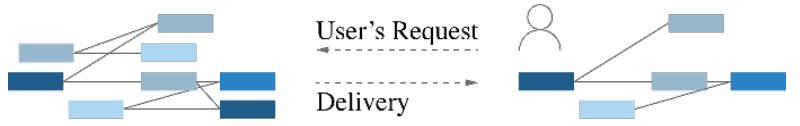

Fig. 2 Scheme of a filtering process triggered by a user request.

\subsubsection{Implementation using Validity Rules}

In Ontolis, we defined a set of Validity Rules that were able to include all elements of the ontology, namely classes, relationships, attributes and instances. Within these rules, all elements could be combined with logical operators such as AND, OR, XOR, and NOT, allowing more complex dependencies to be defined between them.

\subsection{Level 2: semantic transformation}

According to Roser [15], a semantic transformation specifies a transformation between two ontologies, a source ontology (SO) and a target ontology (TO).
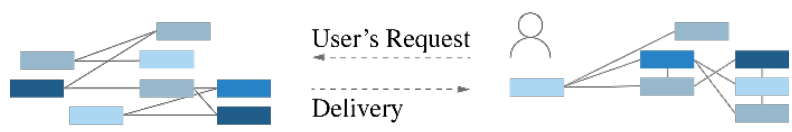

Fig. 3 Scheme of a semantic transformation triggered by a user request.

Through semantic transformation, the elements of a SO can be transformed into another structure (see Figure 3) taking advantage of logical ontology patterns. These ontology patterns consist of links that indicate which elements of the SO need to be transformed into which elements of the TO. If they are equivalent to the elements in the SO, those elements are transformed into the same ones in the TO. If they differ from the elements in the SO, this results in a TO composed of different elements [15]. These links can be reflected in meta ontology, which defines a way of storage, tracing the links between input elements in SO and target elements in TO [16].

\subsubsection{Implementation using QIRA}

The mechanism described in section 3.2 was implemented using $Q I R A$, an ontolis-specific query and transformation language. Building on this, we defined a set of declarative transformation rules to cause some modifications in a target ontology depending on queries. Since the current state of transformations is stored within these rules, QIRA can be understood as a meta ontology. The semantic transformation rules were initialized by user inputs. In our use case, users could select which specific smart device they wanted to receive information from. The resulting (TO) provided them with information that matched the query and the use case. Since the TO was completely transformed, it differed from its "origin" both regarding the number of elements and the structure.

To perform semantic transformations and to enable efficient query answering, QIRA uses an in-house developed engine that imitates a reasoner and exploits the functions of SQL queries and XSLT transformations. In general terms, a reasoner (also semantic reasoner or rules engine) is a software that infers logical consequences from explicitly asserted facts in ontologies [17].

Reasoners are key components for working with ontologies and ontology transformations, especially at higher levels of intelligent information access. This is due to the need for correctness and consistency of ontologies, which are essential to obtain correct query results and provide intelligent information. The reasoners verify if there are any inconsistencies and logical contradictions within the ontology [17]. Also, some of the knowledge modeled might not be explicit. In such cases, reasoners can draw conclusions based on inferences to deduce implicit knowledge and derive new facts [18].

In contrast to the previously described levels of intelligent information access (filtering and semantic transformation), the processes on the following levels can be additionally triggered through external events or certain conditions allowing higher automation of information delivery and access processes. Thus, the following levels and their respective retrieval techniques have been revolutionizing the way information is accessed.

\subsection{Level 3: intelligent publication process}

In the context of this paper, an intelligent publication process is defined as the dynamic aggregation and delivery of information in a non-ontological representation (see Figure 4). This means, information is converted into system-independent formats, such as HTML or PDF. As a result, information, or any content, can be provided on diverse domains and media platforms for different types of users. Intelligent publication processes generally build on semantic transformations (see section 3.2). The difference among them is that their results are not just a modified ontology model, but a different format. The ontology is validated and interpreted by a reasoner. Provided that very specific use cases are defined and represented in the ontology model, the reasoner identifies relevant information.
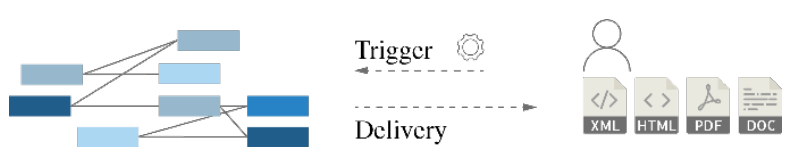

Fig. 4 Scheme of an intelligent publication process triggered by events. 
On level of intelligent publication process, the delivery of content is usually context-, event- or processdriven [3]. According to Ziegler [3] such processes can be defined as push procedures, in which corresponding systems automatically gather information on operating conditions or product states, which can eventually trigger the information aggregation and delivery processes.

\subsubsection{Implementation using the PublicForm}

Based on our semantic transformation rules defined with QIRA, the elements of the ontology were converted to HTML elements upon user requests. Here, QIRA offered some genuine advantages. Particularly, the generic ability to retrieve relevant ontology elements and to redirect the transformation results to the so-called PublicForm. The latter was developed by Ontolis, to support the on-demand delivery of obtained information over the Internet.

We developed an interface that encapsulated the PublicForm and displayed information within a web browser by means of style sheets. Users were provided with read-only access to the information contained in the ontology. In this respect, the web-based interface did not provide explicit functionality for any direct modification of the underlying ontology model. Moreover, users could not see the actual semantic model.

Users were initially presented with four different categories that sought to simulate pre-filters. Selecting a certain category would lead them to the specification of the use case which was then presented as a summary, including the PublicForm. There, users could submit queries that triggered the corresponding ontology transformation. This process recomposed the information and presented the results as a coherent response.

Another means of performing this conversion is to use, for example, Quick Response (QR) codes, Radiofrequency identification (RFID) chips or user locations that can further automate the delivery and access processes driven by ontology transformations. Such automatic conversion processes hold great promise and can be seen as a key for intelligent and advanced methods of accessing information [19]. However, the premise for such approaches is that the relevant information or content should be semantically categorized and precisely described [2], and becomes therefore automatically retrievable, reusable, and reconfigurable [20]. This would also support the idea of micro documents aiming to provide information only to the extent required by users. Ziegler [3, 21] defines micro documents as logic-driven compositions of semantically linked information with limited context, bridging the gap between single topic delivery and monolithic documents. That means that search processes or requests can trigger content aggregation in the form of micro documents. The latter can benefit from additional enrichment of more complex semantic models, such as ontologies.

\subsection{Level 4: intelligent content delivery}

Within the process of intelligent content delivery, information from multiple heterogeneous information sources is aggregated through ontologies (see Figure 5). This can be done by linking the resources to ontologies through relations expressed via mappings. This mapping process is referred to as Database-to-Ontology Mapping. A general definition of this concept could be given as follows: an establishment of correspondences between the source components and the ontology components [22]. For further theoretical discussions on this topic, the reader is referred to Ghawi and Cullot [22] who propose several approaches to enable ontology-based information integration.

The level of intelligent content delivery uses the semantic patterns of ontologies to facilitate information integration across various information sources. During the process, information is extracted, aggregated and presented as a cohesive entity. This is in contrast to the previous levels described above, where information comes from a single source. The advantage of such information integration processes is that already existing information can be accessed and imported from different systems and, if needed, can be seamlessly combined with other information that might not be directly available to users.

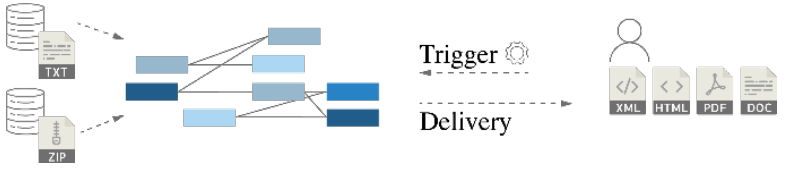

Fig. 5 Scheme of an intelligent content delivery process triggered by events.

\subsubsection{Possible future implementation scenarios}

In future projects, we would like to relate information from various information sources, such as Content Management Systems (CMS), and transform them into a single target form by using ontologies as semantic middleware [3]. Especially in the realm of technical communication, much attention has been paid to CMS, which store, retrieve and use granular (technical) information on end-user products, while enabling singlesourcing mechanisms [23]. In such systems information can be used and flexibly, or even automatically, retrieved during composing activities [24]. This means information is repurposed and reassembled to create a variety of information products addressing different needs.

To bridge the heterogeneity between different information sources (e.g. CMS), the sources must be mapped to ontologies which will express their semantics. In other words, both a source and an ontology need to be semantically related on a conceptual level [22]. This would require first the identification of the sources, which may contain different types of information. Secondly, the information stored in those sources would be unequivocally defined using metadata. From here, the mapping process would start which includes following sequential process steps: a transformation of the source 
schema into the ontology structure followed by a migration of the source components into ontology components. The latter, also referred to as ontology population, can be realized as a query-driven process that transforms the information resulting from a given query [22]. The outlined mapping process is fairly dynamic and starts running as soon as updates are made within the source(s).

The query results can be redirected to web services, such as Content Delivery Portals (CDP). Such portals are increasingly used to enable the interoperability between several and distributed sources. They aim to answer user queries and deliver the right amount of corresponding information [25].

Logical semantic patterns of ontologies can bridge the gap between data integration and migration. This can ultimately foster the efficient reuse of often already existing information from multiple (distributed) information sources. Therefore, building on intelligent content delivery processes and making use of ontology transformations can support the idea of accessing intelligent, semantically enriched information.

\section{Study limitations}

The present paper has certain limitations. First and foremost, both the ontology model (for Smart Homes) and ontology transformations were defined and carried out in Ontolis. Thus, they are limited to the functional capabilities and scope of this tool. However, the validity of the findings described and presented could be extended, for example, by examining the levels of information access in different ontology-editing tools to find any similarities or differences in the conclusions.

Another limitation has to do with the ontology itself. According to Staab and Studer [26], ontologies should express a consensus, that is a shared view between multiple parties rather than a single view. Even though attempts were made to include different perspectives on the domain of Smart Homes, the possibility of relevant omissions and researcher biases remains. Therefore, our recommendation for future research on the same topic would be to develop an ontology model based on use cases and evaluate the ontology by discussing it with experts from the field. This could enable the analysis of even more realistic information access scenarios and ensure real-world application.

\section{Conclusion}

Ontology transformations can process large semantic models effectively and provide personalized and use-case specific information. As for the definition of intelligent information in section 1 , ontology transformations can improve making information truly intelligent. However, the outcome of transformations does not offer the same value among all user groups. On the one hand, end-users only benefit if the results are presented in a media-specific format. Information tagged with standard HTML tags, provides the ability for end-users to simply view and "consume" the aggregated information [27]. In this project, we managed this by starting at the level of intelligent publication process.

Ontology developers and domain experts on the other hand use ontologies to store, effectively organize, share and annotate large sets of information [27] in their fields enabling collaboration in modeling and within companies. They can use ontology transformations to narrow down the domain of interest to a specific information need or to extend the set of information contained within the ontology.

We have described approaches by which the delivery of intelligent information and its access can be enhanced by using ontology transformations. We have shown how information retrieval techniques can be applied as ontology transformations. Depending on the level of implementation, ontology transformations offer in-time and on-demand access to personalized, contextualized information. From this perspective, both ontologies and ontology transformations can be portrayed as very promising approaches of intelligent information access. However, it needs to be considered that the development of use cases, ontologies and ontology transformations is a "process of iterative design [that] will likely continue through the entire lifecycle of the ontology." [28]

We would like to thank the International Office, the Faculty of Information Management and Media, the AStA Students Union, the master's program Communication and Media Management from Karlsruhe University of Applied Sciences for the financial support. We also thank Thomas Weinmann and Christian Koller from Ontolis for great collaboration, insightful discussions, and their support as well as Professor D. Roy from the University of Aizu (UoA) for the organization of the conference at UoA.

\section{References}

1. S. Leukert: Wandel des Kontextbegriffs. In J. Henning, M. Tjarks-Sobhani (Eds.): Intelligente Informationen. Schriften zur Technischen Kommunikation. 22nd ed. Stuttgart: tekom e.V., pp. 103-110. (2017)

2. W. Ziegler: Semantic Information Development for Intelligent Content Delivery. In Official Journal of Japan Technical Communication Association JTCA, pp. 18-29. (2019)

3. W. Ziegler: Drivers of Digital Information Services: Intelligent Information Architectures in Technical Communication. In D. Roy (Ed.): ACM Chapter Proceedings on Educational Technology, Language and Technical Communication. Aizu, Japan: The University of Aizu, pp. 48-53. (2019)

4. T. Nguyen, W. Ziegler: Interactive Content Delivery: Technologies, Use Cases and Industrial Applications for IoT Scenarios. In tekom e.V. (Ed.): Intelligente Informationen. Stuttgart. tcworld conference, pp. 142-144. (2017)

5. C. D. Manning, P. Raghavan, H. Schütze: Introduction to Information Retrieval. Cambridge: Cambridge UP. (2009) 
6. N. Guarino, P. Giaretta: Ontologies and knowledge bases: Towards a terminological clarification. In N. Mars (Ed.): Building and sharing very large-scale knowledge bases. Towards very large knowledge bases. Amsterdam: Ios Pr. (1995)

7. T. R. Gruber: A translation approach to portable ontology specifications. In Knowledge Acquisition 5 (2), pp. 199-220. (1993)

8. S. Spaccapietra: Journal on Data Semantics XI. Berlin: Springer. (2008)

9. Cambridge Dictionary: Transformation $\mid$ meaning in the Cambridge English Dictionary. Cambridge University Press. Available online at https://dictionary.cambridge.org/dictionary/english/t ransformation, checked on 3/5/2020. (2020)

10. O. Šváb-Zamazal, V. Svátek, F. Scharffe, J. David: Detection and Transformation of Ontology Patterns. In A. Fred, J. L. G. Dietz, K. Liu, J. Filipe (Eds.): Communications in computer and information science, Bd. 128, Knowledge Discovery, Knowledge Engineering and Knowledge Management: First International Joint Conference, IC3K. Funchal, Madeira, Portugal, pp. 210-223. (2009)

11. H. Fischer, L. Krägel: Modeling of Complex Metadata in Technical Communication by Ontologies. In D. Roy (Ed.): ACM Chapter Proceedings on Educational Technology, Language and Technical Communiacation. Aizu, Japan: The University of Aizu, pp. 11-16. (2019)

12. A. Cockburn: Writing effective use cases. Boston: Addison-Wesley. (2006)

13. G. Armano, M. Gemmis; Semeraro, Giovanni; Vargiu, Eloisa: Intelligent Information Access. Berlin, Heidelberg: Springer-Verlag Berlin Heidelberg. (2010)

14. N. J. Belkin, W. B. Croft: Information filtering and information retrieval: two sides of the same coin? In Communications of the ACM 35 (12), pp. 29-38. (1992)

15. S. Roser, B. Bauer: Ontology-Based Model Transformation. In J. Bruel (Ed.): Satellite events at the MoDELS 2005 conference. MoDELS 2005 international workshops, Berlin: Springer, pp. 355356. (2006)

16. B. Wouters, M. Gervais: Ontology Transformations. In C. Chi (Ed.): 2012 IEEE 16th International Enterprise Distributed Object Computing Conference (EDOC). pp. 71-80. (2012)

17. S. Abburu: A Survey on Ontology Reasoners and Comparison. In International Journal of Computer Applications (17). (2012)

18. D. Moshman: From inference to reasoning: The construction of rationality. In Thinking \& Reasoning 10 (2), pp. 221-239. (2007)

19. J. Oevermann, W. Ziegler: Automated Classification of Content Components in Technical
Communication. In Computational Intelligence (33). (2017)

20. A. Rockley, C. Cooper, K. Halvorson: Managing enterprise content. A unified content strategy. 2nd ed. Berkeley, CA: New Riders. (2012)

21. W. Ziegler: Extending intelligent content delivery in technical communication by semantics: micro documents and content services. In D. Roy (Ed.): Proceedings of the ETLTC ACM Chapter International Conference. Aizuwakamatsu, Japan. (2020)

22. R. Ghawi, N. Cullot (Eds.): Database-to-Ontology Mapping Generation for Semantic Interoperability. Vienna. Third International Workshop on Database Interoperability. (2007)

23. S. Closs: Single-Source-Publishing. Topicorientierte Strukturierung und DITA. Frankfurt am Main: entwickler.press. (2007)

24. C. Oberle, W. Ziegler: Content Intelligence for Content Management Systems. tcworld. Available online at http://www.tcworld.info/rss/ article/content-intelligence-for-contentmanagement-systems/, updated on 10/2012, checked on $3 / 4 / 2020$. (2012)

25. W. Ziegler: The Evolution of Content Management towards Intelligent Delivery Systems for Technical Communication. In Frontier, Official Journal of Japan Technical Communicators Association JTCA, pp. 68-75. (2017)

26. S. Staab, R. Studer: Handbook on Ontologies. Dordrecht: Springer. (2009)

27. T. E. Potok, M. T. Elmore, J. W. Reed. N. F Samatova: An ontology-based HTML to XML conversion using intelligent agents. In R. H. Sprague (Ed.): Proceedings of the 35th Annual Hawaii International Conference on System Sciences. Los Alamitos, Calif: IEEE Computer Society Press, pp. 1220-1229. (2002)

28. N. F. Noy, D. L. McGuinness: Ontology Development 101: A Guide to Creating Your First Ontology. Stanford, CA: Stanford University. (2001) 